\title{
Analysis of X-rays in bone remodelling around Active and Replace dental implants
}

\author{
Brygida Trochim ${ }^{1} \cdot$ Marta Borowska ${ }^{1}$ (D) Janusz Szarmach²
}

Received: 18 February 2021 / Revised: 10 June 2021 / Accepted: 19 June 2021 / Published online: 1 July 2021

(c) The Author(s) 2021

\begin{abstract}
Purpose of the study: The purpose of the study was to examine on the basis of radiographic images of bone tissue, whether there are differences in the rate of bone remodelling using different shapes of implants in the mandible and maxilla. Moreover, the study also compares texture features obtained on the basis of these images for healthy bone tissue, bone directly after implantation and after a 12-month period of prosthetic loading. Materials and Methods: The subject of the analysis was radiovisiogram images obtained from the Medical University of Bialystok from the Department of Dental Surgery. They are radiovisiogram photographs of 146 people aged 18-74, treated implantally due to missing teeth. The whole group of patients received two types of implants (Active and Replace) of the same company, made of titanium, intraosseous, screw-in. Results: It has been shown that both in the upper jaw and the mandible, the values of texture parameters obtained for bone images made after one year of prosthetic loading are closer to healthy tissue than immediately after implantation. These values for the mandible were relatively closer to those obtained on the basis of healthy tissue than those for the upper jaw. The bone around the implant with a single threading achieved better results in the mandible than the one with a double threading. Conclusion: The type of bone tissue and the shape of the implant have an impact on the achieved osseointegration. With the passage of time and the process of bone remodelling, the damaged tissue returns to its normal structure.
\end{abstract}

Keywords Radiographic image interpretation · Image processing · Computer-assisted · Dental implantation

\section{Introduction}

Implantology is recognized as one of the fastest growing areas of dentistry. The use of implants in the case of missing teeth facilitates maintenance of appropriate anatomical conditions and functioning of the dental system. Currently, various types and shapes of implants are used. Titanium and its alloys are the most commonly used materials due to their biocompatibility $[2,7,9,14,30]$.

Marta Borowska

m.borowska@pb.edu.pl

Brygida Trochim

brygidatrochim@gmail.com

Janusz Szarmach

januszszarmach@gmail.com

1 Institute of Biomedical Engineering, Faculty of Mechanical Engineering, Bialystok University of Technology, Wiejska 45C, 15-351 Białystok, Poland

2 Department of Oral Surgery, Medical University of Bialystok, M. Curie-Skłodowskiej 24A, 15-276 Białystok, Poland
The effectiveness of implantological treatment largely depends on the achieved bone tissue anastomosis with the implant surface (osseointegration). It is a dynamic process in which, inter alia, through biomechanical forces occurring in the implantological system, a balance between bone tissue growing and resorption is maintained $[3,8,13,21,22,24,35]$.

There are many methods of assessing bone and implant connection. One of them, which allows a precise description of changes, is the analysis of radiovisiographic images (RVG). With the use of diagnostic imaging software it is possible to extract the texture features. This enables a detailed assessment of the state of bone tissue, changes occurring during bone healing and achieved osseointegration $[4,31,36]$. A large part of the analysis of osseointegration is based on this method $[8,16,26,27,29]$.

The results of such analysis provide information necessary to apply new implantological methods and solutions that improve the quality of treatment with the use of dental implants. There are studies showing that over time, the bone around the implant rebuilds and returns to its normal structure $[8,16,29]$. Moreover, significant differences in implant 
stabilization between the upper jaw and the mandible were also confirmed [28,32].

The aim of the study was to examine on the basis of radiographic images of bone tissue around the implant, whether there are differences in the rate of bone remodelling in time using different shapes of implants in the mandible and maxilla, compared to healthy tissue.

\section{Materials and methods}

\subsection{Data collection}

The subject of the analysis was RVG images (radiovisiograms) obtained from the Medical University of Bialystok from the Department of Dental Surgery. They are RVG photographs of 146 people aged 18-74, treated implantally due to missing teeth. Written informed consent has been obtained from all the objects involved in the study to publish this paper. The whole group of patients received the same implant system-Nobel Biocare, made of titanium (Ti6Al-4V), intraosseous, screw-in. Radiographic images were performed with KODAK RVG 6100 set with the real resolution over $14 \mathrm{pl} / \mathrm{mm}$. The study protocol was approved by the Local Ethical Committee of Medical University of Bialystok, Poland.

The research material concerns two types of implants: single- (replace implant) and double-threaded (active implant) dental materials possess the ability to compact the bone. The first of them was introduced for 108 patients (51 in the maxillary and 57 in the mandibular bone), while the second for 38 patients (35 in the maxillary and 5 in the mandibular bone). The photographs were not distinguished by gender, as the number of individual groups obtained as a result would be too small to carry out reliable analyses. Images of the implant zones were performed directly after introduction and after 12 months of prosthetic loading (Fig. 1). From the received images, apart from the implant area, areas of healthy, intact bone were used (31 figures from the maxillary and 28 from the mandibular bone). These radiographic images were assigned to the following comparison groups:

- Active-Replace-Healthy bone, II stage: upper jaw;

- Active-Replace-Healthy bone, II stage: mandible;

- I-II stage (Active)—Healthy bone: upper jaw;

- I-II stage (Replace)—Healthy bone: upper jaw;

- I-II stage (Replace)—Healthy bone: mandible.

where stage I means directly after implantation and stage II means a 12-month period of prosthetic loading. The analyzed groups were distinguished in terms of bone, because the jaw and mandible differ in bone structure [6]. A group containing
I-II stage (Active): mandible statements was not created due to insufficient number of samples.

\subsection{Pre-processing}

Preparation of images for analysis was performed in four stages (Fig. 1):

1. rotation of the photograph so that the implant is in a vertical position;

2. excision of the region of interest (ROI) containing the bone around the implant;

3. histogram stretching;

4. histogram equalization.

All these operations were carried out in ImageJ, the intended program for image processing and analysis. For all RVG images, rectangular regions of interest with similar dimensions were selected on both sides of the implant. Based on the literature, a bilinear interpolation method was used for the rotation [33,37]. Histogram operations were used to attempt improve the quality digitized image $[17,20]$. The images after processing in ImageJ were then analyzed in the Qmazda program. No normalization was performed with this program. As a result of actions in this program, almost 600 texture features values were extracted on the basis of ROI of each image.

\subsection{Statistical analysis}

Statistical analysis was performed in the Statistica programme. Significant differences in means were examined by analysis of variance, the selection of which was based on checking the normality of distributions and homogeneity of variance of parameters. The post hoc test was also used to more accurately assess differences between individual subgroups. The Shapiro-Wilk test was performed to check the normality of the parameters distribution of each subgroup. As a result of this test, it was found that in most of them there were no significant differences from the normal distribution (level $p<0.05$ ). For these parameters, the Brown-Forsyth test was performed, which showed that there were no significant differences in the homogeneity of the variance of features in subgroups. In order to verify the significance between means, one-way ANOVA (analysis of variance) was selected. Tukey's honest significant difference (HSD) test was used as the post hoc test.

From among the parameters for which the ANOVA test showed significant differences between the subgroups, those regarding the brightness component and those with the lowest $p$ level were chosen. 
Fig. 1 Stages of RVG image processing

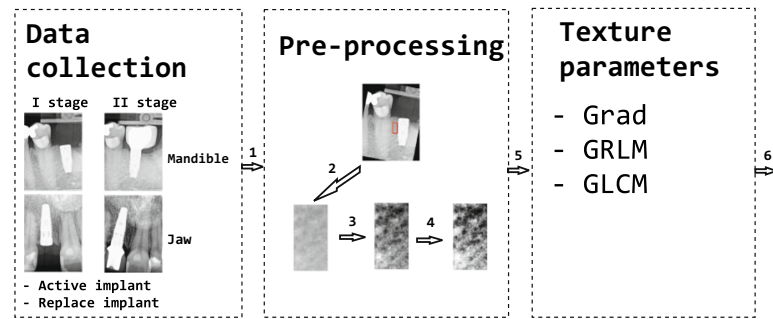

Statistical

analysis

- The Shapiro-Wilk test

- The Brown-Forsyth test

- One-way ANOVA anlysis

- Tukey's honest significant difference (HSD) test

\subsection{Texture analysis}

The parameters selected for analysis concerned gradient map, run-length matrix and grey-level co-occurrence matrix.

\subsubsection{Gradient map}

The gradient map describes the differences between the brightness of pairs of adjacent points or at a given distance in vertical and horizontal directions. On the basis of the gradient matrix, it is possible to determine the basic statistical parameter of the first order-the average $[11,12,15,19]$.

\subsubsection{The run-length matrix}

The run-length matrix describes how often in a given ROI particular lengths of points of the same brightness appear. The number of columns is the largest possible string length for the analyzed direction, and the number of rows determines the number of brightness levels. Based on this matrix, it is possible to obtain parameters such as reverse moment of short string emphasis, moment of long string emphasis, heterogeneity of brightness levels, heterogeneity of string length and the share of image surface in strings [10-12,15,38].

\subsubsection{The grey-level co-occurrence matrix}

The grey-level co-occurrence matrix consists of elements describing the frequency of pairs of pixels of a given brightness and neighbourhood. Based on this matrix energy, contrast, correlation, summation average, summation and differential variance, and entropy and differential entropy are calculated [5,10-12,23,38].

\section{Results}

All parameters analyzed below refer to the horizontal direction of analysis and are characterized in their subgroups by normal distribution and homogeneity of variance. Analysis of variance (ANOVA) showed that for each of them there are statistically significant differences between at least two subgroups.
Table 1 The mean values and standard deviations (mean \pm SD) for group: I-II stage (Active)—Healthy bone: upper jaw

\begin{tabular}{llll}
\hline Feature & $\begin{array}{l}\text { I stage } \\
N=66\end{array}$ & $\begin{array}{l}\text { II stage } \\
N=66\end{array}$ & $\begin{array}{l}\text { Healthy bone } \\
N=31\end{array}$ \\
\hline F1 & $14.70 \pm 3.27$ & $16.27 \pm 3.25$ & $27.56 \pm 5.29$ \\
F2 & $0.56 \pm 0.08$ & $0.58 \pm 0.07$ & $0.73 \pm 0.05$ \\
F3 & $0.41 \pm 0.08$ & $0.45 \pm 0.07$ & $0.61 \pm 0.05$ \\
F4 & $0.44 \pm 0.06$ & $0.41 \pm 0.05$ & $0.29 \pm 0.04$ \\
F5 & $0.71 \pm 0.07$ & $0.74 \pm 0.07$ & $0.96 \pm 0.06$ \\
\hline
\end{tabular}

F1-YD8GradMean

F2-YD5GrlmHFraction

F3-YD5GrlmHMRLNonUni

F4-YD5GlcmH3InvDfMom

F5-YD5GlcmH3DifEntrp

Analysis of variance (ANOVA), $p<0.001$

\subsection{Analysis of bone image texture alteration over time}

On the basis of the post hoc test, significant differences between each subgroup were obtained, for each group relating to the comparison of bone texture changes over time. Moreover, the most significant differences occur between the healthy bone subgroup and the others. This indicates the differences between the texture of bone tissue directly after implantation, after a year and a healthy bone. The average values with standard deviations and $p$ values of the analyzed parameter ANOVA test are shown in Tables 1,2 and 3.

This shows that after a period of one year of prosthetic loading, the bone around Active and Replace implants (both in the jaw and the mandible) has a structure more akin to intact tissue than immediately after implantation. The gradient map parameter (YD8GradMean) describes the rapid changes in image shades. Run-length matrix parameters (YD5GrlmHFraction and YD5GrlmHMRLNonUni) indicate that the largest heterogeneity-the least smooth texture-is characterized by healthy tissue, then stage II, and the tissue in the I stage is the least heterogeneous. Grey-level co-occurrence matrix parameters (YD5GlcmH3InvDfMom and YD5GlcmH3DifEntr) also point to the same dependence. The reason for such results may be the reconstruction of the bone structure after its destruction during implantation. After the implant has been introduced into the bone, along with the 
Table 2 The mean values and standard deviations (mean \pm SD) for group: I-II stage (Replace)—Healthy bone: upper jaw

\begin{tabular}{llll}
\hline Feature & $\begin{array}{l}\text { I stage } \\
N=101\end{array}$ & $\begin{array}{l}\text { II stage } \\
N=101\end{array}$ & $\begin{array}{l}\text { Healthy bone } \\
N=31\end{array}$ \\
\hline F1 & $15.24 \pm 3.31$ & $16.81 \pm 3.43$ & $27.56 \pm 5.29$ \\
F2 & $0.56 \pm 0.07$ & $0.60 \pm 0.07$ & $0.73 \pm 0.05$ \\
F3 & $0.42 \pm 0.07$ & $0.46 \pm 0.07$ & $0.61 \pm 0.05$ \\
F4 & $0.43 \pm 0.05$ & $0.41 \pm 0.05$ & $0.29 \pm 0.04$ \\
F5 & $0.72 \pm 0.07$ & $0.75 \pm 0.07$ & $0.95 \pm 0.06$ \\
\hline
\end{tabular}

F1-YD8GradMean

F2-YD5GrlmHFraction

F3-YD5GrlmHMRLNonUni

F4-YD5GlcmH3InvDfMom

F5-YD5GlcmH3DifEntrp

Analysis of variance (ANOVA), $p<0.001$

Table 3 The mean values and standard deviations (mean \pm SD) for group: I-II stage (Replace)—Healthy bone: mandible

\begin{tabular}{llll}
\hline Feature & $\begin{array}{l}\text { I stage } \\
N=114\end{array}$ & $\begin{array}{l}\text { II stage } \\
N=114\end{array}$ & $\begin{array}{l}\text { Healthy bone } \\
N=28\end{array}$ \\
\hline F1 & $20.35 \pm 3.92$ & $22.00 \pm 4.42$ & $28.97 \pm 5.40$ \\
F2 & $0.65 \pm 0.06$ & $0.67 \pm 0.07$ & $0.74 \pm 0.06$ \\
F3 & $0.51 \pm 0.07$ & $0.53 \pm 0.07$ & $0.61 \pm 0.06$ \\
F4 & $0.37 \pm 0.05$ & $0.35 \pm 0.05$ & $0.29 \pm 0.04$ \\
F5 & $0.79 \pm 0.07$ & $0.82 \pm 0.07$ & $0.94 \pm 0.07$ \\
\hline
\end{tabular}

F1-YD8GradMean

F2-YD5GrlmHFraction

F3-YD5GrlmHMRLNonUni

F4-YD5GlcmH3InvDfMom

F5-YD5GlcmH3DifEntrp.

Analysis of variance (ANOVA), $p<0.001$

remodelling of the jaw and mandible, its structure becomes more variable, heterogeneous, less smooth.

The values of the parameters from Tables 1, 2 and 3 were normalized so that the average values of healthy tissue are $100 \%$ and for the remaining stages they were properly calculated as its percentage content. The data normalized in this way are presented in Tables 4, 5 and 6 .

On the basis of Tables 5 and 6, a graph was created to visualize the comparison of percentage of normalized values of the average of parameters achieved for the upper jaw and mandible for the Replace implant (Fig. 2).

The presented data show in an intelligible way that the mean values of parameters related to bone after 12 months of prosthetic loading are between the values obtained directly after implantation and the healthy tissue. In addition, the values of parameters for the mandible shown in percentage are set closer to $100 \%$ than the jaws'. This dependence occurs for both stages I and II. This is due to differences in the structure between the analyzed bones and the functions they perform. The upper jaw is a pneumatic bone, so its density is
Table 4 Percentage representation of normalized averages for group: I-II stage (Active) - Healthy bone: upper jaw

\begin{tabular}{llll}
\hline Feature & $\begin{array}{l}\text { I stage } \\
(\%)\end{array}$ & $\begin{array}{l}\text { II stage } \\
(\%)\end{array}$ & $\begin{array}{l}\text { Healthy bone } \\
(\%)\end{array}$ \\
\hline F1 & 53.3 & 59 & 100 \\
F2 & 75.7 & 79.7 & 100 \\
F3 & 67.6 & 72.8 & 100 \\
F4 & 152.6 & 144.2 & 100 \\
F5 & 74 & 77.6 & 100 \\
\hline
\end{tabular}

F1-YD8GradMean

F2-YD5GrlmHFraction

F3-YD5GrlmHMRLNonUni

F4-YD5GlcmH3InvDfMom

F5-YD5GlcmH3DifEntrp.

Table 5 Percentage representation of normalized averages for group: I-II stage (Replace)—Healthy bone: upper jaw

\begin{tabular}{llll}
\hline Feature & $\begin{array}{l}\text { I stage } \\
(\%)\end{array}$ & $\begin{array}{l}\text { II stage } \\
(\%)\end{array}$ & $\begin{array}{l}\text { Healthy bone } \\
(\%)\end{array}$ \\
\hline F1 & 55.3 & 61 & 100 \\
F2 & 76.7 & 81.4 & 100 \\
F3 & 69.1 & 74.6 & 100 \\
F4 & 149.8 & 142.8 & 100 \\
F5 & 76 & 79.1 & 100 \\
\hline
\end{tabular}

F1-YD8GradMean

F2-YD5GrlmHFraction

F3-YD5GrlmHMRLNonUni

F4-YD5GlcmH3InvDfMom

F5-YD5GlcmH3DifEntrp

Table 6 Percentage representation of normalized averages for group: I-II stage (Replace)—Healthy bone: mandible

\begin{tabular}{llll}
\hline Feature & $\begin{array}{l}\text { I stage } \\
(\%)\end{array}$ & $\begin{array}{l}\text { II stage } \\
(\%)\end{array}$ & $\begin{array}{l}\text { Healthy bone } \\
{[\%]}\end{array}$ \\
\hline F1 & 70.2 & 75.9 & 100 \\
F2 & 87.9 & 91.5 & 100 \\
F3 & 82.8 & 87.4 & 100 \\
F4 & 128.7 & 122.5 & 100 \\
F5 & 84.8 & 87.8 & 100 \\
\hline
\end{tabular}

F1-YD8GradMean

F2-YD5GrlmHFraction

F3-YD5GrlmHMRLNonUni

F4-YD5GlcmH3InvDfMom

F5-YD5GlcmH3DifEntrp.

lower than the other bones (including the mandible). Thus, the degree of remodelling, depending on the quality of bone tissue, as well as the integration of bones with the implant, achieves better results in the mandible. 
Fig. 2 Percentage representation of normalized average values of Replace implant parameters

\section{0}

[\%]

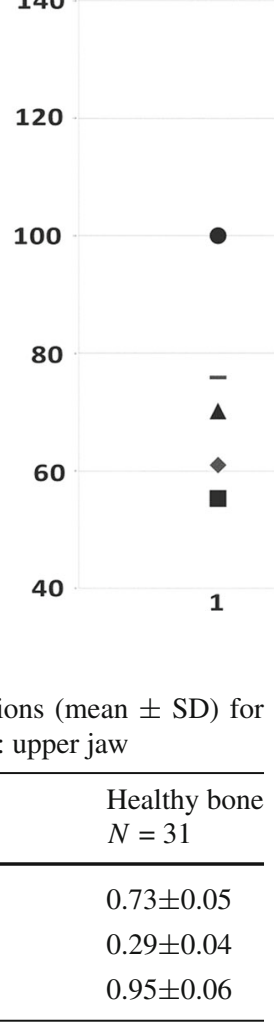

Table 7 The mean values and standard deviations (mean \pm SD) for group: Active-Replace-Healthy bone, II stage: upper jaw

\begin{tabular}{llll}
\hline Feature & Active & $\begin{array}{l}\text { Replace } \\
N=101\end{array}$ & $\begin{array}{l}\text { Healthy bone } \\
N=31\end{array}$ \\
\hline F2 & $0.58 \pm 0.07$ & $0.60 \pm 0.07$ & $0.73 \pm 0.05$ \\
F4 & $0.41 \pm 0.05$ & $0.41 \pm 0.05$ & $0.29 \pm 0.04$ \\
F5 & $0.74 \pm 0.07$ & $0.75 \pm 0.07$ & $0.95 \pm 0.06$ \\
\hline
\end{tabular}

F2-YD5GrlmHFraction,F4-YD5GlcmH3InvDfMom F5-YD5GlcmH3DifEntrp.

Analysis of variance (ANOVA), $p<0.001$

\subsection{Analysis of bone image texture alteration for different implants}

The analysis also included two groups regarding the comparison of healthy tissue and both Active and Replace implants for the second stage, i.e. for bone images taken after one year of prosthetic loading. The first of these groups concerns the upper jaw, and the second one the mandible. As in the previous case, the analysis concerned the horizontal direction. The average values with standard deviations and p-values of the ANOVA test of analyzed parameters are shown in Tables 7 and 8 .

The ANOVA test indicates that for each parameter at least one of the subgroups is significantly different. In the post hoc test it was found that for parameters describing the upper jaw, significant differences occur only between the healthy bone and both types of implants. After a year of prosthetic loading, no significant differences were found between Active and Replace implants in the upper jaw. On the other hand, in the case of the mandible for two parameters (YD8GradMean and YD5GlcmH1DifEntrp), in addition to the above-mentioned differences, statistically significant differences in the mean
Table 8 The mean values and standard deviations (mean \pm SD) for group: Active-Replace-Healthy bone, II stage: mandible

\begin{tabular}{|c|c|c|c|}
\hline Feature & $\begin{array}{l}\text { Active } \\
N=10\end{array}$ & $\begin{array}{l}\text { Replace } \\
N=114\end{array}$ & $\begin{array}{l}\text { Healthy bone } \\
N=28\end{array}$ \\
\hline $\mathrm{F} 1$ & $18.42 \pm 2.50$ & $22.00 \pm 4.42$ & $28.97 \pm 5.40$ \\
\hline F5 & $0.48 \pm 0.04$ & $0.55 \pm 0.08$ & $0.64 \pm 0.09$ \\
\hline F6 & $380.41 \pm 103.52$ & $478.41 \pm 103.52$ & $617.15 \pm 196.21$ \\
\hline
\end{tabular}

F1-YD8GradMean

F5-YD5GlcmH3DifEntrp

F6-YYD8Gab12H6Mag

Analysis of variance (ANOVA), $p<0.001$

between the implant Active and Replace were obtained. The values of these two parameters are characterized by the fact that the average value for the Replace implant lies between the value of the Active Implant and the healthy bone. This means that the type of implant affects the degree of remodelling of the mandible and the Replace implant gave better results here. Larger values of these parameters indicate more rapid shade changes and greater image heterogeneity (implant Replace). Percentage representation of normalized average values (with a healthy bone value of $100 \%$ ) for these two parameters is shown in Table 9. These results are due to differences in implant design. A single threading Replace implant has a more delicate structure, which can determine a better stability in a narrow mandible.

\section{Discussion}

Dental implants have become a common way to replace the missing teeth. Therefore, the best solutions are sought to meet the requirements. One of them is the integration of 
Table 9 Percentage representation of normalized averages for group: Active-Replace-Healthy bone: mandible

\begin{tabular}{llll}
\hline Feature & $\begin{array}{l}\text { Active } \\
(\%)\end{array}$ & $\begin{array}{l}\text { Replace } \\
(\%)\end{array}$ & $\begin{array}{l}\text { Healthy bone } \\
(\%)\end{array}$ \\
\hline F1 & 63.6 & 75.9 & 100 \\
F5 & 75.2 & 85 & 100 \\
\hline
\end{tabular}

F1-YD8GradMean

F5-YD5GlcmH3DifEntrp.

bone tissue with the implant, enabling long-term use of the implant. It is associated with bone remodelling caused by its infraction during the implantation process. The insertion (screwing) of the implant consists in its mechanical connection with the bone tissue. This involves lateral compression of the bone, causing a certain degree of destruction to its structure. Then the bone heals, which takes 6 months, on average [32]. In this study, radiological images were analyzed in the area of implantation of two types of implants. From among almost 600 extracted texture parameters, several repetitive ones were selected, whose values may indicate significant differences occurring in the bone structure during its healing and remodelling. These features were calculated from the algorithms of the autoregressive model, gradient matrix, string length matrix and co-occurrences. Skonieczka in her work also carries out an analysis of these parameters to compare changes occurring in human tissues and distinguishes them as predominant [34]. It has been shown that there are statistically significant differences between the healthy tissue and remodelling time (directly after implantation and after a 12-month period of prosthetic loading). In addition, the values obtained after a year are closer to the healthy bone than those after implantation, which indicates that with time the bone structure around the implant undergoes remodelling. This relationship is constant for the upper jaw and the mandible. It also does not change depending on the type of implant used. Heo et al. proved that after 12 months the bone around the implant has values closer to the bone before surgery than after implantation [16]. The parameter obtained from the gradient matrix (GradMean) describes the rapidity of shade changes. In all groups, the healthy tissue was characterized by the highest heterogeneity of texture, and the smallest heterogeneity was seen in the bone immediately after implantation. Greater image texture uniformity (which means less pixel diversity in the image-more pixels with similar brightness) was obtained for the bones immediately after implantation (Stage I). After the implant has been inserted, the bone tissue around it is 'crushed', which may result in less heterogeneity and higher density. Along with the healing time, normal tissue structures are rebuilt, their density decreases, while heterogeneity increases (trabecular bone) [1]. In a study by Önem et al., based on changes in the frac- tal dimension, they noticed a decrease in bone density after bone healing ( 6 months) [29]. This is also confirmed by the research of Heo et al., in which they prove that directly after the implantation the density increased and then decreased, until after 12 months it reached values similar to the healthy bone [16]. The results of the above analysis show that the texture parameters of the mandible images, both after a year and directly after implantation, reached relatively closer values to the healthy bone than in the case of the upper jaw. This is due to differences in their structure and functions. The mandible is a movable bone and is subjected to greater forces than the upper jaw, which results in an increased response to loads and thus faster reconstruction $[6,18]$. The differences in parameter values for different implants-Active and Replace-were also compared. The type of implants has no major impact on the degree of remodelling of the upper jaw bone. However, in the case of the mandible, differences occur for the individual implants. The Active implant has reached values less similar to the healthy bone tissue. This indicates the impact of the implant shape on osteointegration with the mandible. Replace implants have a more delicate shape, which may be beneficial when used in the mandible due to its narrow crestal bone and different structure [18]. In their studies, Saini and Goyal as well as Oh and Kim also noted better results of implant stabilization in the mandible than in the jaw [28,32]. Mesa et al. showed that there are differences between stabilization achieved in men and women, and depending on age [25]. Their research showed that due to, among others, lower bone mass, the implant's stability in women is lower. Another important factor affecting tissue integration with the implant is the implant geometry (diameter, length, thread pitch). With the increase in the implant dimensions, the contact area of the implant with the bone increases too, which supports implant stabilization [28]. Considering the above relationships, it would be necessary to assess the impact of the patient's sex and age, as well as the implant geometry on the values of the parameters obtained, which could not be done, because the number of individual groups obtained in this way would be too small to conduct reliable analyses. After obtaining a greater number of objects for analysis, such a study could be possible, thanks to which even more accurate analysis results would be obtained.

The main highlights of this study are as follows:

- This is extensive study to compare the performances of many texture features for healthy bone tissue, bone directly after implantation and after a 12-month period of prosthetic loading using different types of implants in the mandible and maxilla in the quantitative analysis;

- The texture features are able to recognize subtle patterns present in the RVG images during remodelling of bone. 
The disadvantages of our findings are:

- More subjects are required to assess the impact of the patient's sex and age, as well as the implant geometry on the values of the parameters obtained;

- More subjects are required to discriminate the classes of RVG images.

Acknowledgements The research was performer as a part of the projects WZ/WM-IIB/1/2020 and was financed with the founds for science from the Polish Ministry of Science and Higher Education.

\section{Declarations}

Ethical approval The study was conducted according to the guidelines of the Guidelines for Good Clinical Practice and approved by the Local Ethical Committee of Medical University of Białystok, Poland (protocol code R-I-002/171/2009 and the date of approval: 30-04-2009).

Conflicts of interest The authors declare that there are no financial or personal relationships with other people or organizations that could inappropriately influence this study.

Open Access This article is licensed under a Creative Commons Attribution 4.0 International License, which permits use, sharing, adaptation, distribution and reproduction in any medium or format, as long as you give appropriate credit to the original author(s) and the source, provide a link to the Creative Commons licence, and indicate if changes were made. The images or other third party material in this article are included in the article's Creative Commons licence, unless indicated otherwise in a credit line to the material. If material is not included in the article's Creative Commons licence and your intended use is not permitted by statutory regulation or exceeds the permitted use, you will need to obtain permission directly from the copyright holder. To view a copy of this licence, visit http://creativecomm ons.org/licenses/by/4.0/.

\section{References}

1. Alghamdi, H.S.: Methods to improve osseointegration of dental implants in low quality (type-iv) bone: an overview. J. Funct. Biomater. 9(1), 7 (2018)

2. Ananth, H., Kundapur, V., Mohammed, H., Anand, M., Amarnath, G., Mankar, S.: A review on biomaterials in dental implantology. Int. J. Biomed. Sci. 11(3), 113 (2015)

3. Annunziata, M., Guida, L.: The effect of titanium surface modifications on dental implant osseointegration. Biomater. Oral Craniomaxillofac. Appl. 17, 62-77 (2015)

4. Atsumi, M., Park, S.H., Wang, H.L.: Methods used to assess implant stability: current status. Int. J. Oral Maxillofac. Implants 22(5) (2007)

5. Azemin, M.Z.C., Tamrin, M.I.M., Hilmi, M.R., Kamal, K.M.: Glcm texture analysis on different color space for pterygium grading. ARPN J. Eng. Appl. Sci. 10(15), 6410-6413 (2015)

6. Baydas, B., Yavuz, I., Dagsuyu, I.M., Bolukbasi, B., Ceylan, I., et al.: An investigation of maxillary and mandibular morphology in different overjet groups. Aust. Orthod. J. 20(1), 11 (2004)

7. Bhagania, M.: Implantology: is it the end of the road for dental specialties? J. Oral Maxillofac. Surg. 67(7), 1575 (2009)
8. Borowska, M., Szarmach, J.: Evaluation of dental implant stability using radiovisiographic characterization and texture analysis. In: International Conference on Information Technologies in Biomedicine, pp. 304-313. Springer (2019)

9. Chun, H.J., Cheong, S.Y., Han, J.H., Heo, S.J., Chung, J.P., Rhyu, I.C., Choi, Y.C., Baik, H.K., Ku, Y., Kim, M.H.: Evaluation of design parameters of osseointegrated dental implants using finite element analysis. J. Oral Rehabil. 29(6), 565-574 (2002)

10. Collewet, G., Strzelecki, M., Mariette, F.: Influence of MRI acquisition protocols and image intensity normalization methods on texture classification. Magn. Reson. Imaging 22(1), 81-91 (2004)

11. Doumou, G., Siddique, M., Tsoumpas, C., Goh, V., Cook, G.J.: The precision of textural analysis in $18 \mathrm{f}$-fdg-pet scans of oesophageal cancer. Eur. Radiol. 25(9), 2805-2812 (2015)

12. García, G., Maiora, J., Tapia, A., De Blas, M.: Evaluation of texture for classification of abdominal aortic aneurysm after endovascular repair. J. Digit. Imaging 25(3), 369-376 (2012)

13. Girejko, G., Borowska, M., Szarmach, J.: Statistical analysis of radiographic textures illustrating healing process after the guided bone regeneration surgery. In: International Conference on Information Technologies in Biomedicine, pp. 217-226. Springer (2018)

14. Grey, E., Harcourt, D., O'sullivan, D., Buchanan, H., Kilpatrick, N.: A qualitative study of patients' motivations and expectations for dental implants. Br Dental J 214(1), E1 (2013)

15. Guggenbuhl, P., Bodic, F., Hamel, L., Baslé, M., Chappard, D.: Texture analysis of $\mathrm{x}$-ray radiographs of iliac bone is correlated with bone micro-ct. Osteoporos. Int. 17(3), 447-454 (2006)

16. Heo, M.S., Park, K.S., Lee, S.S., Choi, S.C., Koak, J.Y., Heo, S.J., Han, C.H., Kim, J.D.: Fractal analysis of mandibular bony healing after orthognathic surgery. Oral Surg. Oral Med. Oral Pathol. Oral Radiol. Endodontol. 94(6), 763-767 (2002)

17. Juliastuti, E., Epsilawati, L., et al.: Image contrast enhancement for film-based dental panoramic radiography. In: 2012 International Conference on System Engineering and Technology (ICSET), pp. 1-5. IEEE (2012)

18. Kakolewska, J., Kuras, M., Sokalski, J., Kulczyk, T.: Use of fractal analysis for bone assessment. Dental Forum 42, 103-106 (2014)

19. Klepaczko, A., Kociński, M., Materka, A.: Quantitative description of $3 \mathrm{~d}$ vascularity images: texture-based approach and its verification through cluster analysis. Pattern Anal. Appl. 14(4), 415-424 (2011)

20. Langarizadeh, M., Mahmud, R., Ramli, A., Napis, S., Beikzadeh, M., Rahman, W.: Improvement of digital mammogram images using histogram equalization, histogram stretching and median filter. J. Med. Eng. Technol. 35(2), 103-108 (2011)

21. Le Guéhennec, L., Soueidan, A., Layrolle, P., Amouriq, Y.: Surface treatments of titanium dental implants for rapid osseointegration. Dent. Mater. 23(7), 844-854 (2007)

22. Maciejewska, I., Nowakowska, J., Bereznowski, Z.: Osteointegration of titanium dental implants: phases of bone healing: a review article. Protet 3, 214-219 (2006)

23. Marchand-Libouban, H., Guillaume, B., Bellaiche, N., Chappard, D.: Texture analysis of computed tomographic images in osteoporotic patients with sinus lift bone graft reconstruction. Clin. Oral Invest. 17(4), 1267-1272 (2013)

24. Mendonça, G., Mendonça, D.B., Aragao, F.J., Cooper, L.F.: Advancing dental implant surface technology-from micron-to nanotopography. Biomaterials 29(28), 3822-3835 (2008)

25. Mesa, F., Muñoz, R., Noguerol, B., Luna, J.D., Galindo, P., O’ Valle, F.: Multivariate study of factors influencing primary dental implant stability. Clin. Oral Implants Res. 19(2), 196-200 (2008)

26. Mundim, M.B., Dias, D.R., Costa, R.M., Leles, C.R., AzevedoMarques, P.M., Ribeiro-Rotta, R.F.: Intraoral radiographs texture analysis for dental implant planning. Comput. Methods Programs Biomed. 136, 89-96 (2016) 
27. Obuchowicz, R., Nurzynska, K., Obuchowicz, B., Urbanik, A., Piórkowski, A.: Caries detection enhancement using texture feature maps of intraoral radiographs. Oral Radiol. 36(3), 275-287 (2020)

28. Oh, J.S., Kim, S.G.: Clinical study of the relationship between implant stability measurements using periotest and osstell mentor and bone quality assessment. Oral Surg. Oral Med. Oral Pathol. Oral Radiol. 113(3), e35-e40 (2012)

29. Önem, E., Baksı, G., Soğur, E.: Changes in the fractal dimension, feret diameter, and lacunarity of mandibular alveolar bone during initial healing of dental implants. Int. J. Oral Maxillofac. Implants 27(5) (2012)

30. Qassadi, W., AlShehri, T., Alshehri, A., Alonazi, K., Aldhayan, I.: Review on dental implantology. Egypt. J. Hosp. Med. 31(5704), 1-9 (2018)

31. Radzewski, R., Osmola, K.: Osseointegration of dental implants in organ transplant patients undergoing chronic immunosuppressive therapy. Implant Dent. 28(5), 447-454 (2019)

32. Saini, G.S., Goyal, M.: Objective assessment of implants stability placed in fresh extraction socket using periotest device. Int. J. Oral Impantol. Clin. Res. 3(2), 67-70 (2012)

33. Scott, G., Imam, M.A., Eifert, A., Freeman, M., Pinskerova, V., Field, R., Skinner, J., Banks, S.A.: Can a total knee arthroplasty be both rotationally unconstrained and anteroposteriorly stabilised? A pulsed fluoroscopic investigation. Bone Joint Res. 5(3), 80-86 (2016)

34. Skonieczka, S.: Analiza tekstury obrazów ultrasonograficznych dla oceny żywotności mieśnia sercowego. Ph.D. thesis (2019)

35. Smeets, R., Stadlinger, B., Schwarz, F., Beck-Broichsitter, B., Jung, O., Precht, C., Kloss, F., Gröbe, A., Heiland, M., Ebker, T.: Impact of dental implant surface modifications on osseointegration. BioMed Res. Int. 2016 (2016)
36. Tyndall, D.A., Brooks, S.L.: Selection criteria for dental implant site imaging: a position paper of the American Academy of oral and maxillofacial radiology. Oral Surg. Oral Med. Oral Pathol. Oral Radiol. Endodontol. 89(5), 630-637 (2000)

37. Wang, Q., Li, L., Zhang, L., Chen, Z., Kang, K.: A novel metal artifact reducing method for cone-beam CT based on three approximately orthogonal projections. Phys. Med. Biol. 58(1), 1 (2012)

38. Zwanenburg, A., Vallières, M., Abdalah, M.A., Aerts, H.J., Andrearczyk, V., Apte, A., Ashrafinia, S., Bakas, S., Beukinga, R.J., Boellaard, R., et al.: The image biomarker standardization initiative: standardized quantitative radiomics for high-throughput image-based phenotyping. Radiology 295(2), 328-338 (2020)

Publisher's Note Springer Nature remains neutral with regard to jurisdictional claims in published maps and institutional affiliations. 